\title{
Quand la polysémie se généralise : le labre, le roitelet et l'euprocte se font "vieux »
}

\section{Les désignations relatives à la nature en langue corse}

When polysemy becomes widespread: the wrasse, the wren and the Corsican brook salamander are getting 'old'. Nature designations in Corsican

Muriel Poli

\section{OpenEdition}

\section{Journals}

Édition électronique

URL : https://journals.openedition.org//bl/1031

DOI : $10.4000 / \mathrm{lbl} .1031$

ISSN : 2727-9383

\section{Éditeur}

Université de Bretagne Occidentale - UBO

\section{Édition imprimée}

Date de publication : 1 juin 2015

Pagination : 27-42

ISBN : 979-10-92331-16-5

ISSN : 1270-2412

\section{Référence électronique}

Muriel Poli, «Quand la polysémie se généralise : le labre, le roitelet et l'euprocte se font « vieux » », La Bretagne Linguistique [En ligne], 19 | 2015, mis en ligne le 01 mai 2021, consulté le 22 mai 2021. URL http://journals.openedition.org//bl/1031 ; DOI : https://doi.org/10.4000/lbl.1031

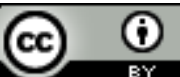

La Bretagne Linguistique est mise à disposition selon les termes de la Licence Creative Commons Attribution 4.0 International. 
Muriel POLI*

\title{
Quand la polysémie se généralise : le labre, le roitelet et l'euprocte se font "vieux ».
} Les désignations relatives à la nature en langue corse

\begin{abstract}
$\mathrm{D}$
ans le cadre du laboratoire Banque de Données Langue Corse (BDLC) ${ }^{1}$ un volume du NALC ${ }^{2}$ consacré aux phytonymes et aux zoonymes a vu le jour en 2009. La thématique «faune et flore» est jusqu'alors assez peu étudiée. Les dialectologues ont traité des noms de plantes et d'animaux en langue corse au fil du vingtième siècle sans toutefois procéder à une étude rendant compte aussi largement des désignations de la nature sauvage et de leur dimension patrimoniale.
\end{abstract}

Dans l'ALEIC, Gino Bottiglioni (1933-1942) enquête sur un réseau relativement important; cependant son questionnaire constitué de 176 questions - soit moitié moins que le nôtre - révèle une faible représentation des plantes du maquis. Son auteur confie d'ailleurs : «Avrebbero potute essere soppresse alcune designazioni di fiori di

* Maître de conférences en linguistique et études corses, membre du laboratoire Banque de Données Langue Corse, polim@univ-corse.fr.

1. www.bdlc.univ-corse.fr.

2. M.-J. DALbera, M. Poli (2009). 
giardino ed aumentate invece quelle dei fiori di campo e di macchia per i quali e denominazioni corse sono ricchissime e varie ${ }^{3} »$.

Le responsaire de l'Atlas linguistique roman relatif à la nature compte également moins de questions. Ces dernières, communes à différents territoires, ne permettent pas toujours de rendre compte de la diversité floristique et faunistique locale. Ainsi, le domaine des animaux semble assez restreint, tout particulièrement la catégorie des oiseaux puisque 18 questions seulement s'y rapportent lorsque notre étude en compte 66 .

Toutefois, à la lecture de ces travaux, nous pouvons nous réjouir de constater que globalement, les faits de langue bien que parfois extrêmement isolés, résistent au temps. Certaines variantes relevées par Bottiglioni sur un seul point d'enquête 70 ans plus tôt, sont corroborées par nos recherches.

Depuis quelques années, d'autres recueils naturalistes ont livré des données dialectales ; citons Marcelle Conrad ${ }^{4}$ (1981), Paul Simonpoli ${ }^{5}$ (1985), Jean-Claude Morati ${ }^{6}$ (1997) ou Petru Casanova ${ }^{7}$ (2010). Pour autant, aucun n'a spécifiquement pour objet l'étude de la dénomination zoologique ou botanique.

Alors que la raison d'être de la terminologie scientifique est de dresser un inventaire aussi complet que possible, la terminologie populaire n'a pas de caractère exhaustif. Les termes de la langue vulgaire sont géographiquement instables. À l'intérieur du domaine d'une même langue, le même nom peut s'appliquer à des espèces différentes selon le lieu; la réciproque est vraie, la même espèce peut avoir des appellations différentes. Elle est foisonnante pour les plantes utiles, absente pour les autres qui n'ont pas besoin d'être identifiées individuellement. Il existe pour elles des génériques très larges : mauvaise herbe, baies sauvages, etc.

3. «On aurait pu supprimer quelques fleurs des jardins au profit des fleurs des champs et du maquis dont les appellations corses sont très riches et variées. "

4. M. ConRAD, Les plantes sauvages dans la vie quotidienne des corses - Essai d'ethnobotanique, Biguglia, Sammarcelli, 1982.

5. P. SimOnPOli (dir.), Arburi, arbe è arbigliule - Savoirs populaires sur les plantes de Corse, Ajaccio, PNRC/Albiana, 1985.

6. J. C. Morati, Topi, topi, topi ... pulenda è porri, Marseille, J. Laffitte, 1999.

7. P. Casanova, Motti, Ajaccio, Piazzola, 2010. 
Le linguiste doit avoir à l'esprit qu'il n'examine pas un corpus basé sur une organisation stable. "Les classifications populaires sont construites sur de nombreux critères sans rapport entre eux : les formes externes ou la taille, les caractères particuliers, les utilisations potentielles, etc. servent à l'identification sans s'insérer dans une systématique descriptive ${ }^{8}$.» On peut cependant, à la manière de Pierre Guiraud ${ }^{9}$, se livrer à un «[...] essai pour saisir les processus de lexicalisation articulés sur une antique préhension du monde, dont les traces sont effectivement inscrites dans la langue et les principes sont effectivement présents dans les modes classificatoires élémentaires de chacun $[\ldots] »$.

\section{Les structures lexicales}

Sur le plan méthodologique, une étude de la faune et de la flore pose un premier problème, notamment dans une zone où la biodiversité est très riche, celui de l'identification des espèces. Si le responsaire de la BDLC offre une trame indispensable, la poursuite de l'étude nécessite d'amener le chercheur à faire sien le questionnaire en multipliant les lectures naturalistes et les prospections in situ auprès d'informateurs et formateurs divers. De même, sur le terrain s'impose à nous le principe selon lequel on ne nomme que les espèces utiles ou nuisibles; c'est la «loi d'intérêt» que mentionne Jean Séguy ${ }^{10}$ (1953). Elle circonscrit, de fait, notre corpus à cet échantillon représentatif.

Pour examiner la manière dont les noms de plantes et d'animaux sont construits et comment ils s'organisent en une nomenclature, on considérera les représentations de la nature qu'ont les locuteurs. Les formes populaires reflètent un processus de dénomination basé respectivement sur les qualités réelles des espèces ainsi que sur la perception qu'on en a. Globalement, cette terminologie est motivée puisque phytonymes et zoonymes recueillis font souvent référence

\footnotetext{
8. R. Nicolä̈, Ethno-taxonomies et représentations étymologiques : en regard des dénominations populaires de la faune, Colloque international «Les zoonymes», Nice, Publications de la Faculté des Lettres, 1997, p. 311-324.

9. P. Guiraud (1986).

10. Page 181.
} 
à la forme, à la couleur, à l'odeur ou au goût, au type de terrain ou à la floraison, à l'usage ou encore aux propriétés des essences. En ce sens, elles révèlent des éléments constitutifs de l'identité d'une communauté et plus largement l'organisation socio-culturelle des peuples.

\section{Différents types motivationnels}

Le corpus recueilli nous permet de mettre en évidence une typologie :

1. Analogie plante-animal

«Parmi les façons de nommer les plantes, l'une des plus fécondes consiste à en assimiler quelque partie (feuille, fleur, épi, racine) à la partie correspondante du corps d'un animal» selon P. Guiraud (1986). Nous avons recueilli plusieurs exemples bâtis sur ce modèle :

- A coda di cavallu ${ }^{11}$, la prêle (Equisetum sp.) généralement nommée ${ }^{12}$ à partir de la forme de son rameau filiforme similaire à la queue d'un cheval (ou d'un renard, ou d'un rat, selon les régions).

- A lingua di boie ${ }^{13}$, le grand plantain (Plantago major) dont la large feuille rappelle la «langue du bœuf».

- L'arechja di porcu, l'arum mange-mouches (Helicodiceros muscivorus ${ }^{14}$ ) est nommé ainsi par similitude entre la fleur poilue de couleur vineuse et l'oreille du cochon. La métaphore est transparente.

\section{Désignations à connotation religieuse}

Elles aussi fonctionnent, en grande partie, par analogie comme l'expose clairement Mario Alinei (1997).

- U cardu cirunaghju, le chardon ${ }^{15}$ précisément Onopordum illyricum, sa grosse tige rappelant un cierge $u$ cirone ; le suffixe

11. Carte 947 du NALC 3.

12. En latin Equisetum, vraisemblablement composé de equus «cheval» et saeta «poil» ou «crinière»; en anglais horsetail, en italien coda di cavallo, en espagnol cola de caballo.

13. Carte 955 du NALC 3.

14. Il s'agit d'une espèce de la famille des araceae menacée et subendémique.

La carte 961 du NALC 3 consacrée à l'arum italicum mentionne arechja di porcu ou arechja purcina.

15. Carte 931 du NALC 3. 
-aghju ${ }^{16}$ aboutit à la forme adjectivale et traduit ici la qualité, la ressemblance.

- U paternostru, le micocoulier (Celtis australis), dont les fruits noirs en grappe, les micocoules, rappellent le chapelet $u$ paternostru.

- A cantamessa, la mante religieuse ${ }^{17}$ (Mantis religiosa) est nommée «chante messe» en raison de sa posture. Elle évoque une nonne, a sora en corse que l'on retrouve également parmi la vingtaine de lemmes répertoriés. Les désignations de la mante religieuse dérivées du latin Cantare sont répertoriées en castillan et dans les Abruzzes ${ }^{18}$.

L'entrée «mante» du TLFi ${ }^{19}$ indique, en exemple tiré de Mérimée ${ }^{20}:$ «(..) J'aurais encore à vous conter celle d'une mante, mantis religiosa - qu'on appelle ici Prega-Diou, prie-Dieu - [...] Elle marche debout sur quatre pattes, sur deux pinces rapprochées sous le menton. C'est pour cela qu'on l'appelle en patois prie-Dieu. »

Les rapprochements avec la religion sont à l'origine de bien d'autres formes populaires locales.

Les données ichtyologiques mises à jour par M.-J. Dalbera et R. Miniconi dans le NALC 2, en 2008, font elles aussi écho à ce phénomène.

La carte de la squille ${ }^{21}$ (Squilla mantis) signale la forme pregadiu mentionnée ci-dessus ; elle pourrait tout autant être rapprochée de la mante religieuse si l'on considère la position de ses pattes.

L'appellation pesciu prete "poisson prêtre » est réservée à l'uranoscope ${ }^{22}$ (Uranoscopus scaber) du grec ouranoskopos «qui regarde le ciel», d'où l'assimilation religieuse.

De la même façon, sur quelques points du réseau littoral, le rocher

16. Suffixe -arium latin.

17. Carte 1057 du NALC 3.

18. Voir la synthèse de P. GARCiA Mouton, Les désignations romanes de la mante religieuse, Atlas linguistique roman, vol. II a, Rome, Istituto Poligrafico e Zecca dello Stato, 2001, p. 257-280.

19. http://atilf.atilf.fr/ (consulté le 11 novembre 2012).

20. Lettres à Mme de la Rochejaquelein, 1870, p. 191.

21. Carte 686 du NALC 2.

22. Carte $624 \mathrm{du}$ NALC 2. 
épineux ${ }^{23}$ (Bolinus brandaris) est désigné capu di Cristu, tête du Christ, par analogie aux épines de la sainte couronne. Comment ne pas faire le lien avec le phytonyme chjoda di Cristu, littéralement «clous du Christ», dénomination du genêt épineux ${ }^{24}$ dans l'extrême Sud de l'île ${ }^{25}$ ?

\section{Désignations phonosymboliques}

Il s'agit de formes à redoublement expressif. Elles concernent essentiellement les insectes et sont vraisemblablement motivées par le battement des ailes ou le bourdonnement, par exemple a barabattula ou a farfalla le papillon ${ }^{26}$ ou encore $u$ zinzalone ${ }^{27}$ la libellule ${ }^{28}$ et quelques autres petits animaux que nous ne citerons pas ici. Ce phénomène concerne également les oiseaux comme en témoignent les désignations $и$ сисси coucou ${ }^{29}$, a turturella tourterelle ${ }^{30}$, a trizina grive draine ${ }^{31}$. Toutes sont formées par onomatopée et reproduisent un chant, un cri, comme l'indique Jean-Philippe Dalbera ${ }^{32}$ ou Michel Contini ${ }^{33}$.

4. Désignations anthropomorphiques

Ces formes, à propos desquelles Jeanine Médélice (1999) précise qu' «il s'agit généralement de métiers liés à la vie en zone rurale - c'est l'univers privilégié des patois - des métiers de tous les jours comme "fileuse", "couturière", "bergère" ou saisonniers

23. Carte $731 \mathrm{du}$ NALC 2.

24. Carte 902 du NALC 3.

25. Nous avions fait le choix de privilégier le générique «genêt épineux» pour ne pas avoir à différencier les différentes espèces de Genisteae, notamment entre calicotome et genista présents en Corse.

26. Carte 1038 du NALC 3.

27. Le site www.etimo.it, évoque, pour la forme zinzala: «[...] imitante il ronzio che fà questo animaletto volando [...]».

28. Carte 1037 du NALC 3.

29. Carte 1106 du NALC 3

30. Carte 1092 du NALC 3

31. Carte 1090 du NALC 3.

32. J. Berns, H. JACOBS, T. SCHEER, Romance Languages and Linguistic Theory, Selected papers from «Going romance», Nice, 2009, p. 103.

33. M. CONTINI, «Les phonosymbolismes : continuité d'une motivation primaire?», Travaux de linguistique 2/2009 ( $\mathrm{n}^{\circ}$ 59), p. 77-103. [en ligne] URL: www.cairn. info/revue-travaux-de-linguistique-2009-2-page-77.htm (consulté le 15 novembre 2012). 
comme "moissonneuse", "vigneronne" ou plus rares comme "médecin"...», évoquent d'autres univers dans notre corpus. La police, l'église ou le domaine militaire sont privilégiés à travers les dénominations : a giandarma le pinson ${ }^{34}$ (Fringilla sp.), u prete le prêtre, a sora la libellule, a capurala la pie grièche ${ }^{35}$ (Lanius $s p$.), etc.

D'autres créations résultent, par induction métonymique, des propriétés de quelques outils liés aux activités humaines. Sur ce modèle, nous pouvons citer : a paghjola «le chaudron »> la mésange ${ }^{36}$ (Parus sp.), l'oiseau et l'ustensile ayant en commun la couleur brune/noire. Paghjola coexiste avec le nom capipaghjulaghja; ici la calotte - u capu - noire de la mésange est directement nommée. Cette motivation est reprise dans une autre variante a carbunaghjola, de carbone «charbon» en lien manifeste avec la couleur noire.

Dans la forme a stacciafarina employée pour désigner le papillon ${ }^{37}$, on discerne le verbe staccià «tamiser» et le nom farina «farine». Les ailes poudreuses de l'insecte laissent effectivement tomber de la poudre au moindre $\operatorname{choc}^{38}$.

La désignation a bugnaghjola la mésange, traduit «celle qui fréquente la ruche» - u bugnu - en raison de ses habitudes alimentaires, les abeilles constituant l'essentiel de son régime alimentaire. D'autres formes composées sur bugnu de type verbe+nom, telles que spizzicabugna ou sgherzicabugna sont consignées dans le NALC 3, elles véhiculent l'idée «donner des coups de bec sur la ruche pour détacher ou attraper un insecte».

Notons que les désignations anthropomorphiques propres à la langue de la mer renvoient elles aussi aux métiers de tous les jours

34. Carte 1076 du NALC 3.
35. Carte 1085 du NALC 3.
36. Carte 1078 du NALC 3.
37. Carte 1038 du NALC 3.

38. M. Contini, «Les désignations romanes de 'papillon'», Atlas linguistique roman, vol. II b, Rome, Istituto Poligrafico e Zecca dello Stato, 2010, p. 179213. «Parmi les caractéristiques physiques du papillon, la poussière des ailes est à l'origine de nombreuses désignations où elle est assimilée, selon les régions à la farine ou au fromage [...] L'association poussière des ailes/farine est présente surtout dans l'aire italienne.» 
mentionnés précédemment. Le forgeron $u$ stazzunaghju et le charbonnier $u$ carbunaru désignent la castagnole ${ }^{39}$, poisson de couleur sombre (cf. infra) alors que $u$ mulinaru le meunier, semble indiquer la couleur claire du ventre du poisson, par analogie à farine blanche.

5. Le sémantisme du «tacheté» et du «sauvage»

Les travaux de Pierre Guiraud (1986) sur la structure du lexique français indiquent que «[...] parmi les différents sémantismes d'où les animaux tirent leur nom, l'un des plus importants et des plus féconds $[\ldots]$ est celui qui les désigne à partir de quelque particularité physique : bécasse, rouge-gorge, huppe [...] et parmi ces caractères (...) l'un des plus fréquents consiste dans les tâches, raies ou bigarrures».

La structure sémantique de notre corpus révèle notamment les adjectifs turcatu, rughjinu, collatu, ochjatu et pichjinatu, variulatu, frisgiatu, maculatu. On y discerne entre autres les étymons latins Robus, Varius, Macula. Parallèlement, les formations collatu et ochjatu délivrent un double message puisque la marque caractéristique sur le plumage ou le pelage, est localisée sur le «cou» u collu ou autour des "yeux» l'ochji ${ }^{40}$. U culombu collatu (ou cullaratu) désigne le pigeon ramier ${ }^{41}$ Columba palumbus et $u$ topu ochjatu le lérot Eliomys quercinus. L'adjectif ochjatu est employé pour différencier les animaux d'un troupeau, en fonction des particularités de leur pelage. Una capra ochjata est une chèvre dont l'œil est entouré de couleur. Ochjata employé comme substantif, exprime la taille prépondérante des yeux et désigne alors l'oblade ${ }^{42}$ Oblada melanura.

Les désignations du «sauvage» peuvent être construites à partir du suffixe -astru du latin -aster. On sait qu'ajouté à un nom de plante il indique son état sauvage, comme oleus olivier > oleaster olivier sauvage. Le corse s'y conforme puisque l'on trouve alivu olivier $>$ agliastru olivier sauvage, sorbu sorbier > surbastru sorbier des

39. Carte $607 \mathrm{du}$ NALC 2.

40. Du latin Oculus.

41. Carte 1096 du NALC 3.

42. Carte 595 du NALC 2. 
oiseleurs ${ }^{43}$, menta menthe $>$ mintrastu menthe sauvage ${ }^{44}$, etc. Dans le même ordre d'idée, le suffixe -astru est, en corse comme en latin, utilisé comme marque de parenté indirecte dans figliastru «beaufils» sur Filius latin, par exemple.

Les adjectifs salvaticu «sauvage», bastardu «bâtard» et leurs antonymes mansu «domestique» ou ghjentile «commun», existent mais sont régulièrement concurrencés par d'autres appellations composées à partir d'un qualificatif d'origine animale. Les formes caninu, tupinu, purcinu, cavallinu, sumerinu, vulpina ${ }^{45}$ semblent largement répandues dans l'espace roman. Giulio Paulis ${ }^{46}$ (1992) mentionne ce système en Sardaigne lorsqu'il commente : "Il carattere selvatico della specie è sottolineato dal referimento ad alcuni animali: cavallo, bue. » Jean Séguy ${ }^{47}$ (1953) l'évoque également à propos des phytonymes gascons : «Le procédé 'plante de tel animal' est un calque traditionnel [...] autrefois assez fécond [...] valeur péjorative de ces mots auxiliaires. » On notera que ce type se rapporte essentiellement à des plantes toxiques ou épineuses donc dépréciées comme $a$ cipolla canina «oignon de chien» (Pancratium illyricum), l'urticule sumerine «orties d'âne» (Urtica sp.), u tangu purcinu «ronce de cochon» (Crataegus monogyna). Parfois le lien entre la plante et ces auxiliaires semble d'un autre ordre comme l'indique Paulis à propos de l'adjectif sumerino ${ }^{48}$. L'auteur explique que le rapport entre le chardon et l'âne peut traduire une connotation péjorative ou indiquer que l'animal recherche le chardon pour sa consommation. Selon certains de nos informateurs, le qualificatif purcinu pourrait également revêtir le sens «qui sert de clôture pour les porcs».

Peut-on parler d'une remotivation moderne?

43. Carte 887 du NALC 3.

44. Carte 906 du NALC 3.

45. De cane « chien», topu « rat», porcu « cochon », cavallu « cheval», sumere « âne » et volpe « renard».

46. G. Paulis, I nomi popolari delle piante in Sardegna, Etimologia Storia Tradizioni, Sassari, Carlo Delfino editore, 1992.

47. Page 282.

48. "Altrettanto comune è il referimento di questi cardi agli animali, sia in senso spregiativo sia perché si tratta di specie effettivamente appetite e ricercate da essi.» G. PAUlis, op. cit., 1992, p. 71. 
Nous pourrions prolonger la liste des classes motivationnelles mises à jour par nos recherches mais il nous semble opportun, à l'invitation des collègues brestois, de poursuivre la réflexion par une mise en perspective entre les noms de la nature terrestre et quelques ichtyonymes communs à la Méditerranée et aux côtes atlantiques.

\section{La démarche sémasiologique}

L'examen des différents atlas linguistiques (ALE, ALiR, atlas régionaux, etc.) permet de comparer les motifs des données lexicales de plusieurs domaines linguistiques de l'Europe. Il devient possible de pointer des similarités récurrentes concernant les motivations à la base de plusieurs dénominations distinctes.

Des phytonymes et zoonymes traduisent le transfert d'un domaine sur un autre : le même signifiant désignant aussi bien un oiseau, un petit animal, qu'un poisson ou même un végétal ! On l'a vu précédemment pour les dérivés de Oculus.

Ce transfert peut découler de la ressemblance de certains traits morphologiques et/ou comportementaux entre les espèces ou bien

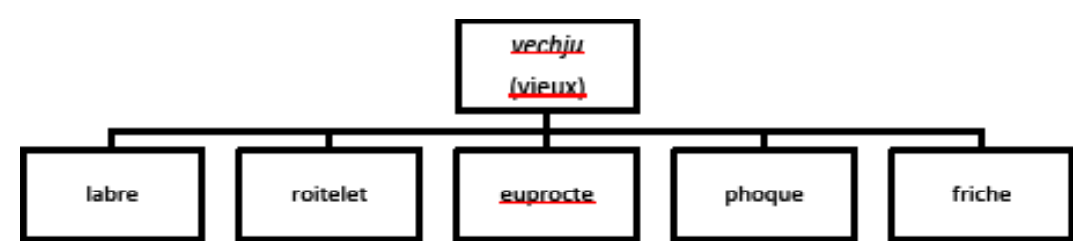

d'un tabou linguistique, les deux pouvant se combiner ; comme en témoignent les exemples originaux exposés ci-dessous.

On sait que l'aspect des espèces est souvent à l'origine de leur appellation. L'entrée «vieille» du CNRTL ${ }^{49}$ mentionne dans la définition : «Nom vernaculaire du labre, probablement en raison de l'aspect ridé de la tête de ce poisson». En corse aussi, a vechja la «vieille», désigne le labre vert (Labrus viridis) ${ }^{50}$ probablement pour

49. www.cnrtl.fr (consulté le 12 novembre 2012).

50. Carte 611 du NALC 3. 
la même raison alors même que, pour la plupart des locuteurs, le motif est oublié.

Le roitelet, oiseau malingre, souvent confondu avec le troglodyte, est localement désigné vichjarella; hypocoristique obtenu par ajout du diminutif courant -ella, précédé d'un premier élément suffixal -ar. Notons que l'oiseau répond également à l'appellation scrizzula ${ }^{51}$ qui véhicule le même signifié «chétif». Les ornithologues ${ }^{52}$ expliquent : «[...] corps de taille moyenne mais la queue et les ailes sont si courtes qu'il apparaît vraiment petit [...]». On songe à l'aspect d'une personne d'âge mûr qui se serait tassée.

La forme $u$ vechjottu, l'euprocte ${ }^{53}$, rappelle la précédente dans sa construction puisque il s'agit d'un terme hypocoristique, cette fois masculin, construit à partir du suffixe diminutif -ottu. On perçoit une note affectueuse dans la désignation de cet animal inoffensif dont les déplacements au sol manquent d'aisance. Plus que son apparence physique, il semblerait que ce soient les manières indolentes ${ }^{54}$ de l'euprocte qui expliquent qu'il soit ainsi nommé.

Notons également le syntagme vechju marinu, littéralement «vieux de mer», donné au phoque moine ${ }^{55}$; il constitue un énième cas d'anthropomorphisation sur la base de "vieux». Les manières pataudes du phoque pour se mouvoir à terre (cf. infra) pourraient être à l'origine de cette variante très largement répandue en corse mais aussi dans le domaine italo-roman. Faut-il plutôt envisager les hypothèses de Mario Alinei (1997) pour qui l'évocation de la «vieille» représente l'ancêtre protecteur renvoyant à une vision magico-religieuse du monde animal? Ou bien s'agit-il simplement d'un continuateur du latin Vitulus comme l'indiquent Dalbera et Dalbera Stefanaggi (2003)?

51. Scrizzula se dit d'une personne particulièrement menue.

52. J.-C. Thibault, Connaître les oiseaux de Corse - Acelli di Corsica, Ajaccio, PNRC/Albiana, 2006.

53. Amphibien endémique corse, nommé plus communément tarantella.

54. À rapprocher de la synthèse des désignations romanes de la salamandre de Annalisa NESI, dans laquelle elle évoque, parmi d'autres hypothèses reliant l'amphibien au monde magique, la donnée comportementale à l'origine de la forme catellu lurcu attestée en corse : « [...] la lenteur des mouvements propre à la salamandre [...]» (ALiR, vol. II.a., p. 479).

55. Carte 806 du NALC 2, Monachus monachus. 
Charles Videgain (2003) analyse les transformations de zahar "vieux» en basque, et parmi elles zakar ${ }^{56}$ dont les diverses acceptions « $[\ldots]$ ont pour sème commun celui de /caduc, ancien, reste/». Ces observations nous amènent à considérer la forme corse vichjetu, construit sur vechju $+-e t u^{57}$ et son signifié «terre en friches » et à la raccorder à notre champ sémasiologique.

Autre exemple, celui de cappuccinu «capuchon» exposé ci-dessous. Il est significatif car il mêle également phytonymes, ichtyonymes et zoonymes. Tous offrent une représentation spécifique du capuce, du latin Caput et Caputium (diminutif). Le TLFi

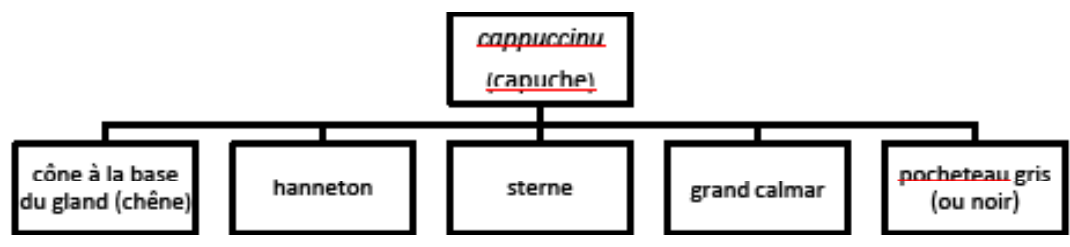

précise «capuchon taillé en pointe que portent certains moines»; la «tête» générant la notion de « coiffe».

Lorsqu'il s'agit de l'" organe écailleux ou épineux, creusé en forme de petite coupe, entourant la fleur, puis le fruit de certains arbres» (TLFi), par exemple pour le chêne, u cappuccinu $^{58}$ désigne précisément la cupule ${ }^{59}$. Le gland est en quelque sorte encapuchonné par la cupule. La carte 869 du NALC 3 s'y rapporte. Elle évoque les métaphores autour de «capuchon», «pipe», «dé»... sans doute liées à des jeux d'enfants.

Chez les coléoptères, en plus de leur couleur noire distinctive, l'idée de «capuchon» est présente si l'on considère leur morphologie caractéristique. Ils possèdent un thorax souvent bombé et scindé par rapport à la tête et à l'abdomen, similaire à une cape, une capuche. Cette particularité physique du hanneton et du scarabée explique la

56. Acceptions : «ordures, résidus, vieilles affaires sans intérêt, croûte sur la peau, quelqu'un de santé ébranlée».

57. Suffixe abondant dans la toponymie.

58. Formé sur la base latine Capa + suffixe diminutif courant -inu.

59. Du bas latin Cupula «petit tonneau de bois». 
forme frate cappuccinu soit «moine encapuchonné». Le scarabée jouit d'autres désignations anthropomorphiques en rapport avec des entités religieuses en occitan, en frioulan et dans les parlers de l'Italie septentrionale.

En ce qui concerne la sterne, sa calotte noire fait office de capuchon. D'autres ornithonymes expriment l'idée de "coiffe», «capuche» ou bien "chapeau». La tête noire (ou contrastée) de la mésange a induit la désignation a cappellina ( $\mathrm{cf}$ infra). La variante cappusgiula ${ }^{60}$ est employée à la fois pour la huppe et la coulemelle. La crête caractéristique de la huppe et le grand chapeau ovoïde du champignon sont à l'origine de la dénomination.

Enfin, toujours parmi les zoonymes construits à partir des noms des parties anatomiques (corporèmes), le grand calmar et le pocheteau à la forme de "capuchon taillé en pointe», enrichissent la dimension sémantique du schéma de départ en y associant les espèces aquatiques.

Un dernier exemple met en évidence des connexions entre les espèces. Comme les deux exemples précédents, il demande à être

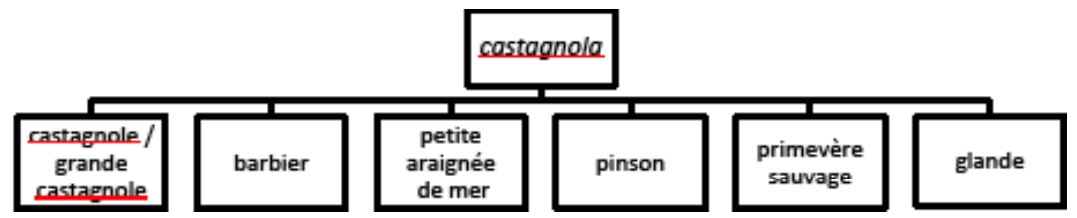

clarifié à partir de la dimension cognitive du langage. Nous tentons de déchiffrer le signe linguistique castagnola "petite châtaigne» en tenant compte des représentations mentales individuelles et/ou collectives des locuteurs.

Ainsi, hormis la forme «primevère», la motivation commune à ces espèces réside dans leur caractéristique physique. Elles arborent toutes la même tonalité brune-rougeâtre. C'est le cas de la castagnole

60. Du latin vulgaire Cappa. 


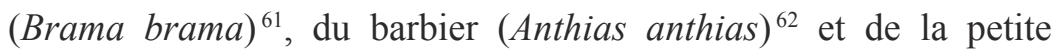
araignée de mer (Maja crispata) ${ }^{63}$.

Le pinson des arbres (Fringilla sp.) au plumage coloré présente une poitrine brun-noisette. La couleur dominante est donc proche du castagnu «châtain» exprimé ci-dessus.

Lorsque castagnola renvoie à la primevère, c'est l'habitat qui semble être dévoilé dans le sens de "fleur qui pousse sous les châtaigniers ». Effectivement, cette plante, peu commune, apprécie particulièrement les sols humides des châtaigneraies de la façade Nord-Est de la Corse, microrégion nommée Castagniccia.

La forme castagnola avec le sens de "glande»-recueillie lors d'une campagne d'enquêtes sur le thème de l'élevage du cochon et de la fabrication de la charcuterie ${ }^{64}$ - semble se référer à la glande thyroïde. Marie-José Dalbera Stefanaggi indique qu'il s'agit d'une «forme métaphorique plus opaque, jamais encore attestée dans les dictionnaires corses dans ce contexte [...]». Ici l'aspect, petit et rond aboutit à l'assimilation entre le fruit et le renflement de la glande.

En définitive, lorsque l'on considère la représentation cartographique des données contenues dans le NALC 3 , on note une proportion très mince de cartes mononymes. Elles concernent presque exclusivement le domaine de la forêt et les noms d'arbres en particulier, en majorité simples calques du latin du type : frassu $>$ Fraxinus, carpine $>$ Carpinus, olmu $>$ Ulmus etc. tandis que le maquis et les animaux produisent un grand nombre de types lexicaux motivés par des «saillances» réelles ou supposées.

Manifestement, la langue de la mer répond à la même tendance puisque sur plus de 300 représentations de poissons et animaux marins divers rassemblés dans le NALC 2 , moins de $15 \%$ des cartes sont uniformes.

61. Carte 582 du NALC 2.

62. Cartes 564 et 607 du NALC 2.

63. Carte 692 du NALC 2.

64. M.-J. Dalbera Stefanaggi (dir.), Purcelli è maghjali, coll. Detti è usi di paesi, éd. Piazzola, Ajaccio, 2006. 


\section{Conclusion}

Le domaine de la faune et de la flore fait une large place à l'imaginaire populaire, offrant ainsi une variation lexicale extrêmement riche. Pour l'ensemble des variantes recueillies, le sens, mais aussi leur structure (dérivation, composition, etc.) ou leur organisation dans une nomenclature dévoilent la manière dont la nature est pensée. Celui qui nomme fait autant appel à l'aspect descriptif qu'aux croyances traditionnelles. L'examen des phytonymes et des zoonymes implique que l'on pénètre les traditions populaires. Sur cette base, de nombreuses perspectives s'offrent aux linguistes.

Le matériau livré par les différents atlas ${ }^{65}$ facilite l'examen transversal des matrices communes à la langue de la nature, posant comme préalable de raviver le lien motivationnel souvent altéré grâce notamment au soutien de l'étymologie.

En ces temps où les institutions publiques entendent donner un nouvel élan à la politique linguistique et redynamiser la pratique de la langue corse dans tous les domaines de la société, prenons garde car on sait que «la planification linguistique se raidit en face de la variation ${ }^{66} »$. Si la Corse devait accéder à un statut institutionnel plus officiel, elle est à considérer dans toute sa richesse, au titre de la variété des possibilités de choix qu'elle offre au législateur.

\section{Bibliographie}

ALINEI M., «Magico-religious motivations in european dialectes : A contribution to Archaeolinguistiques », Dialectologica et Geolin-

65. Dans le cadre de cadre de cette communication, nous retiendrons principalement le Nouvel Atlas Linguistique et ethnographique de la Corse (NALC) et l'Atlas Linguistique Roman (ALiR).

66. J.-B. MARCELLESI, Quelle Politique linguistique pour quel État-nation?, Glottopol, Revue de sociolinguistique en ligne, $\mathrm{n}^{\circ}$, janvier 2003, p. 158 [en ligne] URL : http://glottopol.univ-rouen.fr/numero_1.html (consulté le 26 octobre 2012). 
guistica, $\mathrm{n}^{\circ}$ 5, 1997, p. 3-30.

Atlas linguistique roman, vol. II.a et II.b, Istituto Poligrafico e Zecca Dello Stato, Roma, 1996-2009.

BotTiglioni G., Atlante Linguistico Etnografico Italiano della Corsica, Pisa, 1933-1942.

CONTINI M., «Les phonosymbolismes : continuité d'une motivation primaire ? », Travaux de linguistique 2, n 59, 2009, p. 77-103.

DALBERA J.-Ph., «Dimension diatopique, ressort motivationnel et étymologie. À propos des dénominations romanes de l'orvet», Quaderni di Semantica, XVIII/2, 1997, p. 195-213.

DAlbera J.-Ph., DAlbera StefanagGi M.-J., «La petite bête qui monte, qui monte... La dimension motivationnelle dans la dénomination du "petit". Étymologie et reconstruction lexicale», Parole romanze, Alessandria, éd. Dell’Orso, 2003.

Dalbera M.-J., MiniCONi R., Nouvel Atlas Linguistique de la Corse. Le lexique de la mer, vol. II, Paris/Ajaccio, éd. CTHS/éd. Piazzola, 2008.

DAlBera M.-J., POLI M., Nouvel Atlas Linguistique et ethnographique de la Corse. Flore et faune, vol. III, Paris/Ajaccio, éd. CTHS/ éd. Piazzola, 2009.

GUIRAUD P., Structures étymologiques du lexique français, Paris, Larousse, 1986.

MÉDÉLICE J., "Sur quelques anthropomorphismes liés à l'activité humaine dans les désignations de la faune et la flore sauvages», Géolinguistique, n 8, 1999, p. 51-81.

PaUlis G., I nomi popolari delle piante in Sardegna, Sassari, éd. Carlo Delfino, 1992.

SÉGUY J., Les noms populaires des plantes dans les Pyrénées centrales, Barcelone, Monagrafias del Institute de Estudios pirenaicos, 1953.

VIDEGAIN Ch., «Vieillir en basque et zahar», dans Actes du colloque Les mots du vieillir, Paris, Institut Universitaire de France, 2003, p. 100-107. 